We can thus explain the failures which have been reported with small lamps. Trillat's method of generating the gas by driving it out of its solution by heating the latter under pressure in an autoclave, avoids the difficulty by setting free the gas very quickly.

Formic aldehyde $\left(\mathrm{CH}_{2} \mathrm{O}\right)$ is a gas under ordinary conditions, but it may be changed into a solid form. This is called paraformaldehyde or tri-oxymethylen. Its formula is $\left(\mathrm{CH}_{2} \mathrm{O}\right)_{3}$ and it may be considered as condensed formaldehyde. It is an imperfectly crystalline substance which is volatilized by heat. The density of the gas thus formed shows that the complex molecule has been decomposed and the simple one, $\mathrm{CH}_{2} \mathrm{O}$, formaldehyde, formed. This method of generating the gas directly from the solid, which takes place at a not high temperature, is likely to prove the most practical means of producing the disinfectant without the use of expensive apparatus as in Trillat's method.

\section{A GLANCE AT SOME RELATIONS OF DEN- TISTRY TO GENERAL MEDICINE.}

\footnotetext{
Presented to the Section on Stomatology at the Forty-eighth Annual Meeting of the American Medical Association, held at Philadelphia, Pa., June 1-4, 1897.

BY GEO. F. EAMES, M.D., D.D.S. BOS'TON, MAss.
}

The average dentist not only fails to recognize many indications which call for systemic treatment, but fails to see his need of such recognition. He still jumbles medical terms. His practice is largely empirical. He sees in part, but acts as one comprehending all; he gives advice to his fellow practitioners more freely than he would if he knew more.

If a new drug is advertised he tries it a few times, and immediately writes an article on it. He treats all forms of pyorrhea by scraping the teeth, but the deposit continues to accumulate, and the scraping is repeated. He treats sensitive dentine by the application of obtundents, and never dreams of prophylaxis or the correction of the underlying cause.

The teeth under certain conditions decay with great rapidity, and extra dollars are received for extra plugs inserted. The teeth are stained and eroded, only to be met with more plugging, and the mechanical removal of the stain.

During a persistent and.alarming hemorrhage from the socket of a tooth, the heart is allowed to go on thumping against the chest wall, thereby resisting the local styptic, and tending to increase the hemorrhage.

It is still true, in too many cases, that a tooth is extracted solely because the patient asks that it be done. Many dentists still ask the patient what kind of filling they will have inserted, little thinking that if in certain cases the request is complied with the dentist may be sued for malpractice.

The dental college with its required three years' course and Latin entrance examinations, still continues to let loose thousands of graduates whose spelling and use of ordinary English is excruciating, and whose understanding of the principles of pathology and medicine is practically nil. These go out to practice on humanity.

Shortly after I had begun to study dentistry, I was introduced by my preceptor to a very prominent minister who said, "so this young man is going to learn to haul teeth!" My preceptor wisely answered, "that, among other things." Those "other things" have engaged my attention ever since, and there are and ever will be, other things to follow, for if there is one characteristic of the marvelous advance which dentistry as a profession has made, it is the great number of new relations which have been discovered to exist, not only between it and other branches of medicine and surgery, but relations with the arts and sciences.

It has been said that the dentist's daily work is largely mechanical; many make it so; many see only the mechanical side of it; for example, an operator may be engaged in plugging a large distal cavity with gold, but may not realize the fact that in some cases of nervous susceptibility, the entire organism may sympathize with the local irritation.thus produced, to the extent of shock, or nervous collapse. What is the position of the mechanical operator in such a case? In his fail. ure to recognize the vital connection of the teeth with the human organism he has failed to discharge his full duty to the patient, and is guilty of criminal neglect.

A dentist may be called upon at any time to exercise his medical knowledge immediately, in cases of nervous spasm, hysteria, syncope, shock, collapse, foreign bodies swallowed, poisoning, hemorrhage, etc.; or in a more leisurely way in the consideration of questions relating to stomatitis, adenoid vegetations, malignant growths, operations during pregnancy, and the various inflammatory conditions of the dental pulp and pericementum.

But there are other fields which have not been so fully explored, and which are vital to the interests of our profession. Teeth are being lost at an alarming rate on account of the ravages of pyorrhea alveolaris, so called, and this fact alone calls for deep medical research, for a thorough physical examination, including the blood, the ealivary and renal secretions, followed by appropriate treatment.

In some of my recent cases the personal history has shown no indications of disease, when an examination of the secretions has shown conclusively the existence of abnormal conditions. This shows the necessity of a thorough examination, without regard to the patient's answers or knowledge of his condition.

In order to do this a most thorough study of the theory and practice of medicine is absolutely required. I can see no other way to meet these pathologic conditions in the mouth, believing as I do that they are the expression of some diathesis or other general disturbance. As medical specialists we are facing the serious problem of saving thousands of teeth which at the present time are being lost in spite of the great advances which our profession has made, and it rests with us, as members of the representative medical body of the United States, to use every means in our power to secure the requirement of a broader general and medical education as one of the essential conditions of graduation. If this be accomplished, the holder is sure of recognition in any medical organization, and the dental profession will stand for one of the most profound and philanthropic agents of the healing art.

Eye Protector-The Journal d'Hygiène of March 10 describes an extension for the eye-glasses of bicyclers, etc., made of celluloid or some similar substance, which clamps over the eye-glass and extends to enclose and fit tight over the entire orbital region, preventing the entrance of dust or wind, although ventilated at the side. 